\title{
Automatic Tap Control System in the Smart Home using Android and Arduino
}

\author{
Vani K.S. \\ Assistant Professor \\ Department of Computer Science \& Engineering \\ Acharya Institute of Technology \\ Bangalore
}

\author{
Shrinidhi P.C. \\ Department of Computer Science \& Engineering \\ Acharya Institute of Technology \\ Bangalore
}

\begin{abstract}
In this busy world everybody are interested in making their routine works automated and also want to monitor the elderly people and patients at home. One of the routine works at home is Plant watering and is very important when the people leave the home for vacation or emergency as the plants may end up drying due to lack of water. Helping the elderly and disabled people to control the taps for their daily activities is also a challenging job. This system is about Tap control control system using the smart phones and the arduino boards. This can be used to control the taps for plant watering and other taps in the home through internet by sitting anywhere in the world. Arduino 2560 board with Ethernet Shield is used in this project. Arduino is coded using arduino codes and the app development is done using Android programming.
\end{abstract}

\section{Keywords}

Arduino Board, Ethernet shield, Android, Elderly People, Plant Watering, Smart home, Solenoid Valve

\section{INTRODUCTION}

In this competitive world everyone are busy in their official works and other personal works so that they don't have sufficient time to complete other works at home such as plant watering and other works. Everybody is also interested in making their work automated.

Automation of the home, housework or household activity is called Home automation. People may be interested in control of lighting, control of air conditioner and heating appliances, security locks of gates and doors and other systems. Home automation helps to provide improved convenience, comfort, energy efficiency and security. Home automation plays a vital role in the life of the elderly and disabled. They can be more independent and lead good quality of life who might otherwise require caregivers or institutional care.

With the popularity and familiarity of smart phones and tablets among common people the concept of home automation has been increased greatly in recent years and also giving a great relief to the caregivers. The integration of Internet of Things with Home Automation has added to the functionality of Home Automation

The main motivation for this project is to give the elderly people, handicapped and patients a safe and independent living by providing the tap automation in the smart home .In this project we are also showing how the taps present in the home, office, shops etc. can be controlled through internet by the android mobile devices using arduino board and other electronic devices. A low cost smart home environment for remotely monitoring and helping the elderly people and patients in their everyday life activities is presented here.

\section{RELATED WORK}

Android is the mobile operating system developed by Google and based on Linux kernel. Android is designed mainly for smart phones and tablets. Android applications are becoming more popular nowadays in all areas of life. Android Smart phones and Android applications are specially being designed for home automation and to take care of elderly people and patients. Accelerometers, gyroscopes and proximity sensors are adding an added advantage to the smart phones where the caregivers of the elderly people, patients and pets can be relaxed as they will be notified about the all the events associated with the elderly people. In this project Android SDK is used along with Eclipse IDE. Now Android has released an official IDE called Android Studio for Android application development.

Arduino is an open source prototyping platform with easy to use hardware boards and software. User can give the input to the arduino boards through many sensors and through internet messages. These inputs can be used to take some action and trigger the events either offline or online. The open source Arduino software IDE makes it easy to write the codes and upload it to the arduino board.

Arduino 2560 (Fig 2.1) which is a microcontroller based board on ATMega 2560 is used in this project. This board is commonly recommended for 3D Printers and Robotics Projects. Along with Arduino 2560 Ethernet shield is also used which helps to connect the internet and control the watering system in the home by sitting anywhere in the world. Ethernet shield is connected to the network by using the RJ45 connector. The Ethernet shield also has a reset controller which helps the ethernet shield to reset on every power up operation.

An automatic plant watering system is created in [3]. This project uses Arduino board, which consists of ATmega328 Microcontroller. It is programmed in such a way that it will sense the moisture level of the plants and supply the water if required. This type of system is often used for general plant care, as part of caring for small and large gardens. Normally, the plants need to be watered twice daily, morning and evening. So, the microcontroller has to be coded to water the plants in the greenhouse about two times per day. plant more self-sufficient, watering itself from a large water tank and providing itself with artificial sunlight. The prototype reports status of its current conditions and also reminds the user to refill the water tank. The system automation is designed to be assistive to the user.

A smart home system to control the lighting, temperature and to detect the intrusions and smoke and fire accidents is presented in [1]. Arduino Board with Ethernet shield has been for monitoring the people at home. This monitoring system continuously monitors the elderly people at home and in case 
of emergency emails is sent to the caregivers and a alarm is also generated.

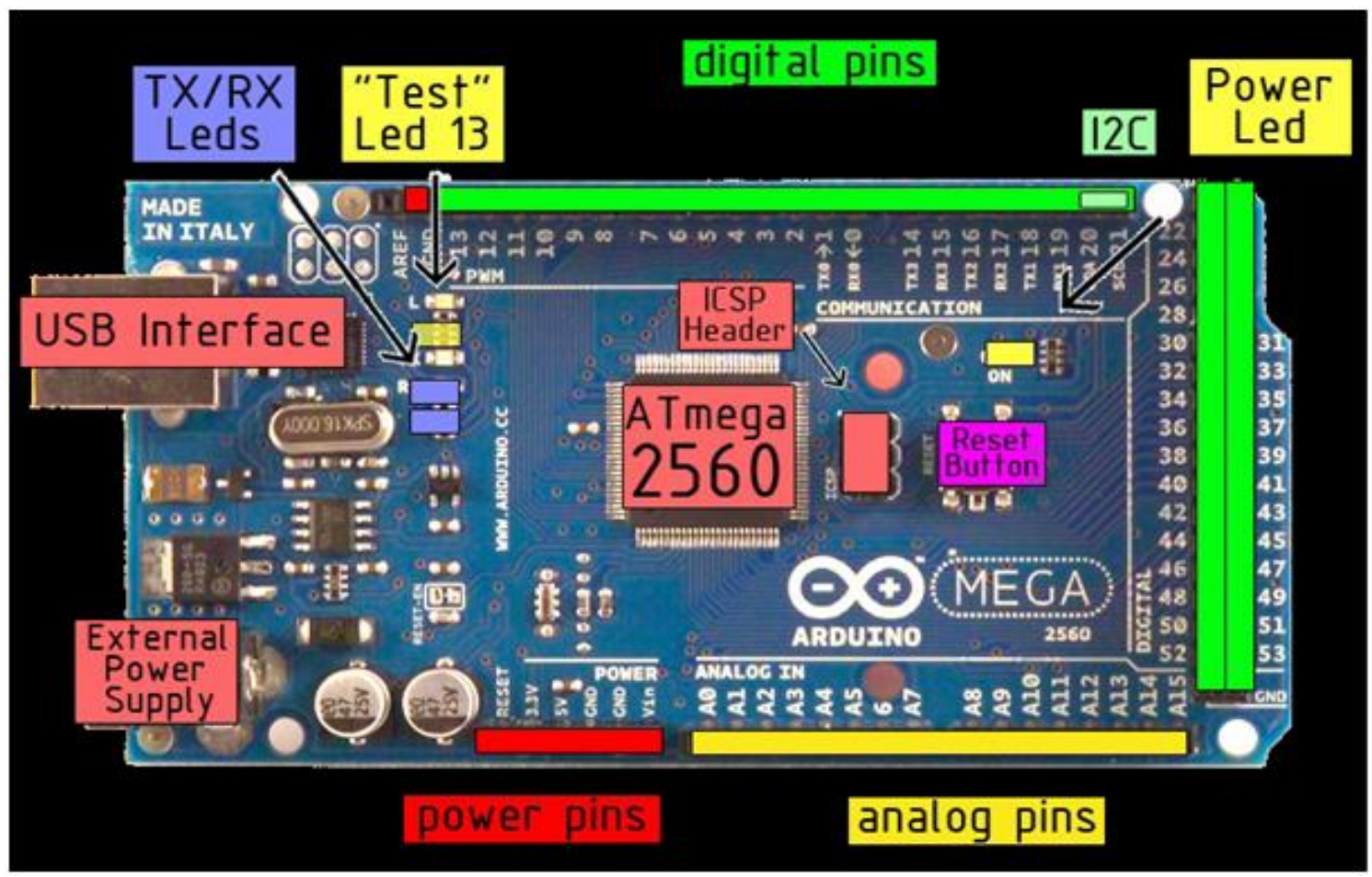

Fig 2.1: Arduino Atmega 2560

The main aim of [4] is to provide automatic irrigation to the plants which helps in saving money and water. The entire system is controlled using 8051 micro controller which is programmed as giving the interrupt signal to the sprinkler. Temperature sensor and humidity sensor are connected to internal ports of micro controller via comparator, Whenever there is a change in temperature and humidity of the surroundings these sensors senses the change in temperature and humidity and gives an interrupt signal to the microcontroller and thus the sprinkler is activated.

These papers are on automatic plant watering method. At a specified time interval water was fed into the pots by sensing the moisture level of the plants. In addition to watering the plants remotely by using a Smartphone and internet connection we have also implemented the tap control in kitchen and bathroom for the safe living of the elderly people and patients.

Remote controlling of house hold appliances and remote monitoring of elderly people activities at home is presented in [2]. The system is based on Microcontroller MIKRO C software. Intrusion Detection and Human motion detection is also implemented in [2]. Surveillance cameras deployed for security can also be controlled by using Arduino and Ethernet shield. The controlling of the house hold devices is done through the Android Smart phone.

\section{SYSTEM DESIGN}

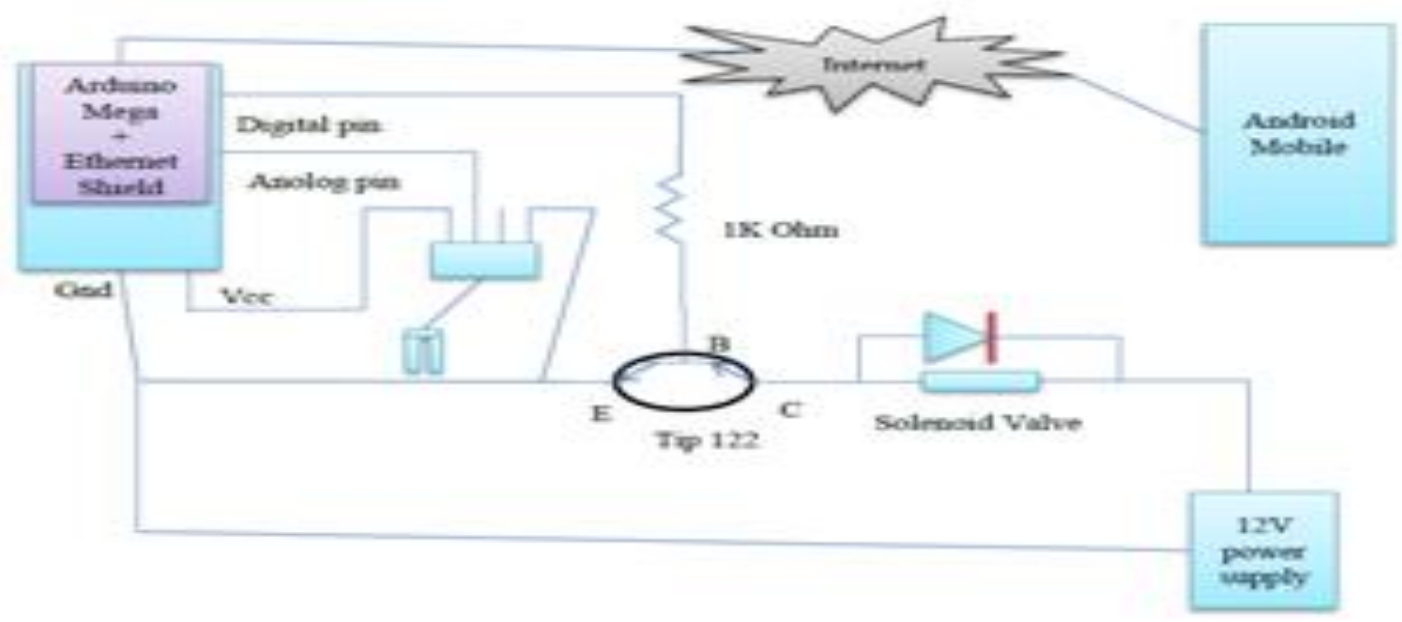

Figure 3.1: System Architecture 
The above diagram is the circuit connection for the tap control through arduino board and the Ethernet shield. The circuit basically consists of Arduino Mega 2560 board, Arduino Ethernet shield, Solenoid valve, Tip 122 transistor, Register of $1 \mathrm{k}$ and a diode. The arduino mega 2560 board is used to control and monitor the sensors, electronic devices and other home appliances through different analog and digital input and output pins.

The arduino board supports many shields such as Wi-Fi shield, Ethernet shield, Bluetooth shield etc. In the circuit diagram the Ethernet shield has been used because the main aim is to control the taps through mobile via internet. The main purpose of Ethernet shield is that it acts as a micro web server. Using the Ethernet shield Internet of Things (IoTs) are been implemented. Ethernet shield acts as an interface between the Arduino board and internet.

The solenoid valve is used in order to control the water flow which acts as the electrically controlled tap. The power supply to the solenoid valve is controlled by the TIP 122 transistor. This helps in the conservation of water as the water flow is controlled via mobile from different places. Initially arduino mega 2560 board and arduino Ethernet shield has been connected to the internet through LAN which in turn is connected to the android mobile. Solenoid valve and diode are connected in parallel to avoid back current. If the authentication to the system is successfully done, the user proceeds with selecting the area like kitchen, bathroom, toilet etc. where they wish to control or monitor. The user can select any of the area to be controlled like garden, kitchen, room etc. Then the status of the tap in the area selected by the user is known by sending request to the arduino. According to the reply the tap status in the application is updated. If the user selects the area as garden then the reply for the request about the current status will also contain the moisture status of the soil where the moisture sensor is implemented. User has to do the necessary changes according to their requirements, then the request will be sent to the arduino. If the operation is done successfully, the success message will be displayed and the corresponding status will be updated in the application. If the requested operation will fail, the failure message will be displayed and accordingly the status will be updated. If the user request for the change of password option then the user has to enter the current password and the new password which will be sent to the arduino. If the entered current password is correct then the new password will be updated in the in the EEPROM.

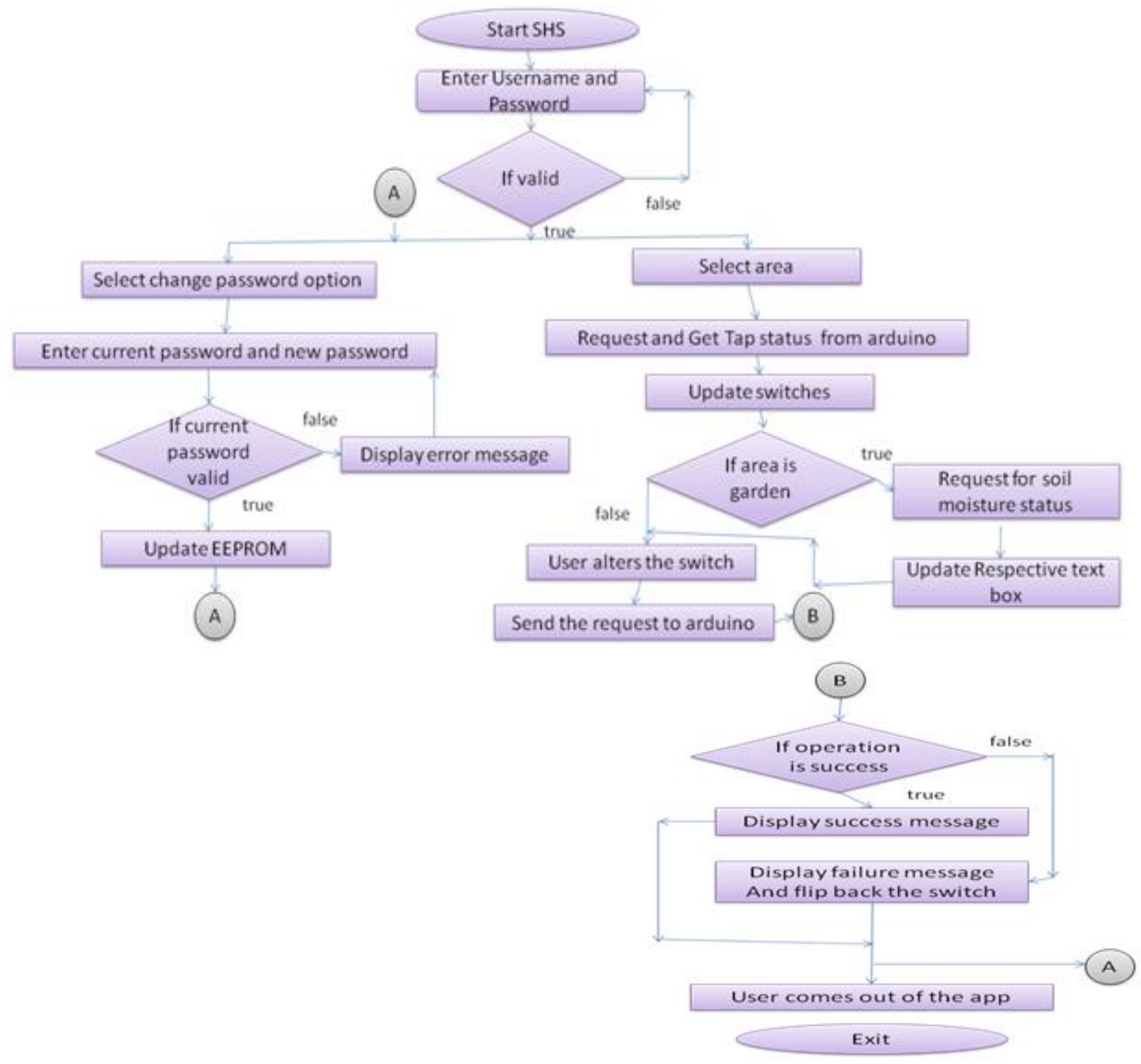

Fig 3.2 Data Flow Diagram 


\section{RESULTS AND DISCUSSIONS}

All the components required for the project were connected as shown in (Fig 4.1). With too many wires the entire system looked complicated and the complexity of entire system was reduced by plastering the wires and the components on a wooden ply board sheet. On top of Arduino Mega 2560 board Arduino Ethernet shield was fixed and the digital pin of arduino was connected to the TIP 122 Transistor. Solenoid valve was also connected to the system according to the design shown above. Android Codes for the SHS app is mainly coded using Eclipse IDE with SDK and ADT installed. Android Development Toolkit is a plug-in with eclipse IDE which can be used to develop android apps.
Once the app was developed the app was uploaded on the Smartphone. When the app was run in the mobile the authentication requirements were displayed and proper authentication input will drive us into the Tap Automation System. This Authentication details will be preloaded in the EPROM (Electrically Programmable Read Only Memory). Authentication details help us to allow the intended person only to control the taps. In the first screen the display of different rooms of the smart home will be displayed(Fig 4.4). After selecting the appropriate room the display of different taps in the selected room will be displayed(Fig 4.2 \& Fig 4.3). This system along with the camera based video surveillance system helps to monitor the requirements of elderly people and patients and help them to switch ON/OFF the taps when required.

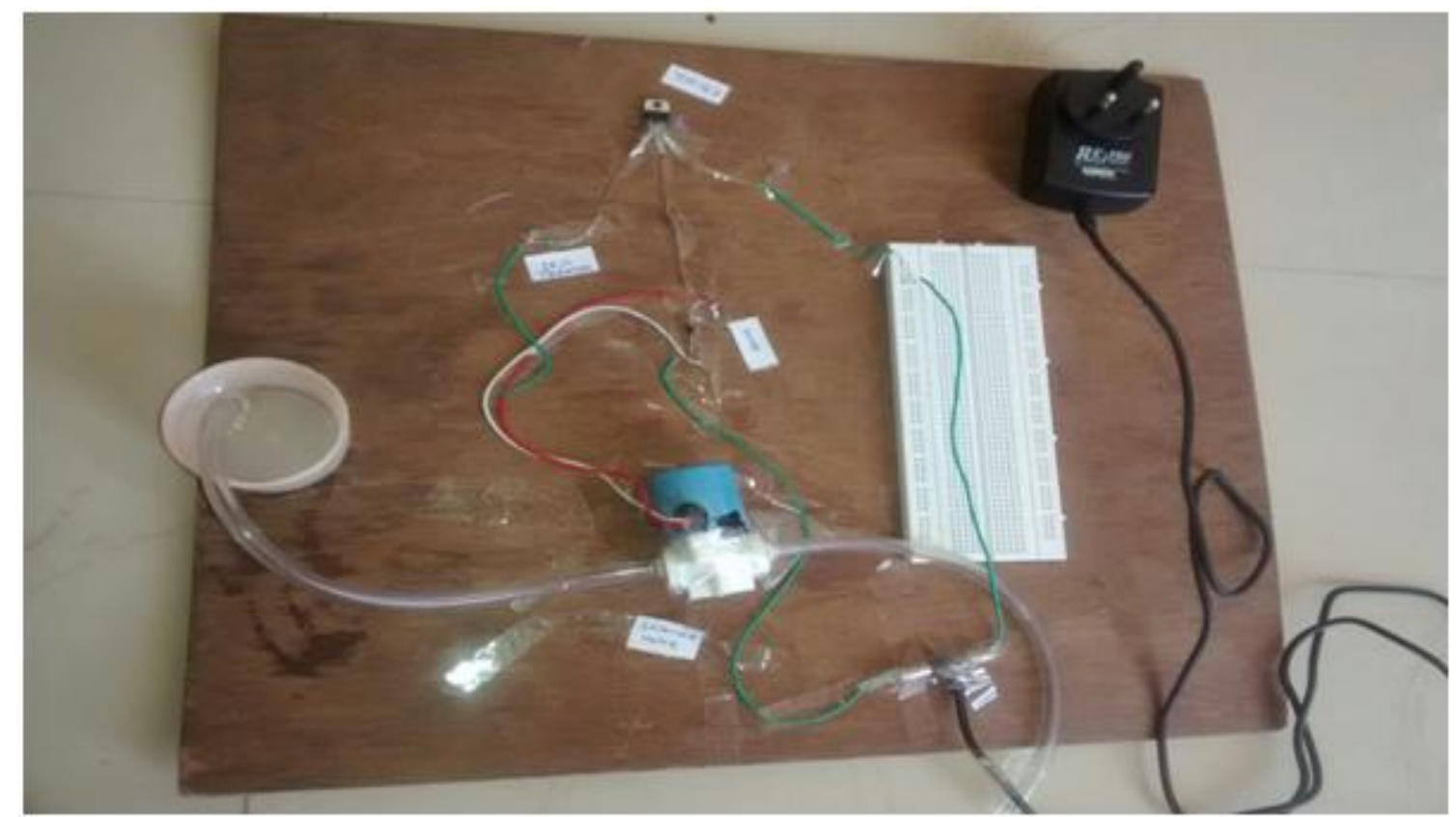

Fig 4.1 : Components and the layout of the project

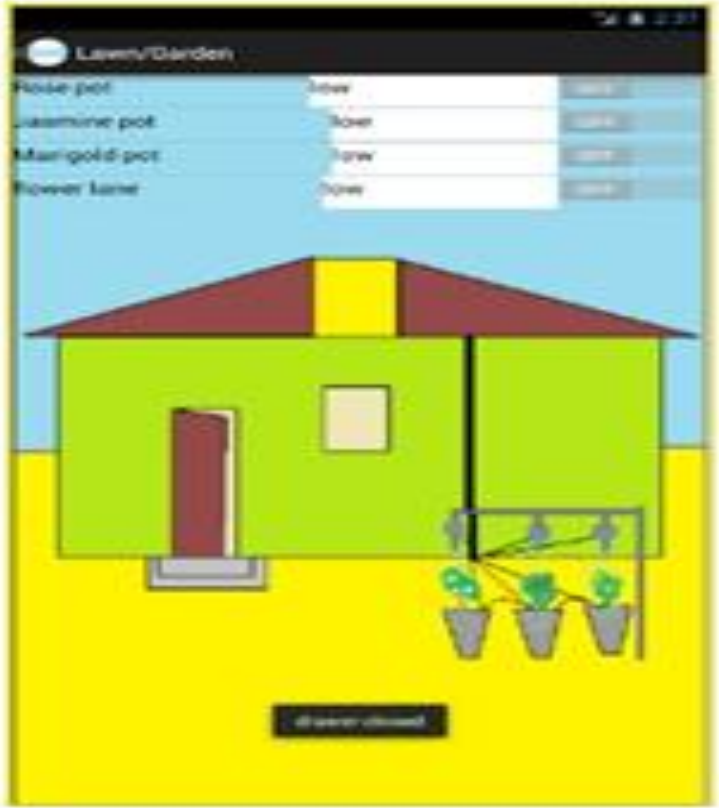

Fig 4.2: After selecting the garden area

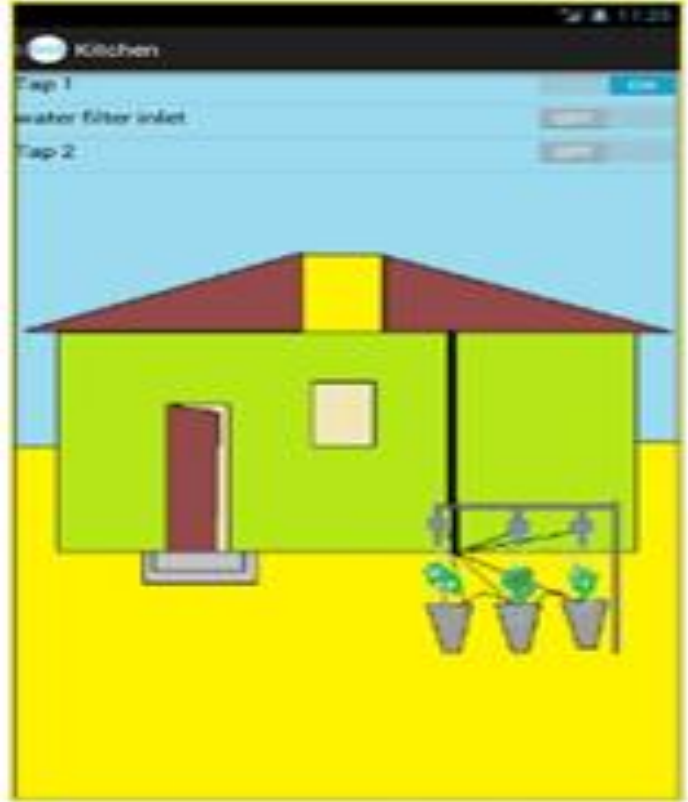

Fig 4.3: After selecting the Kitchen Area 


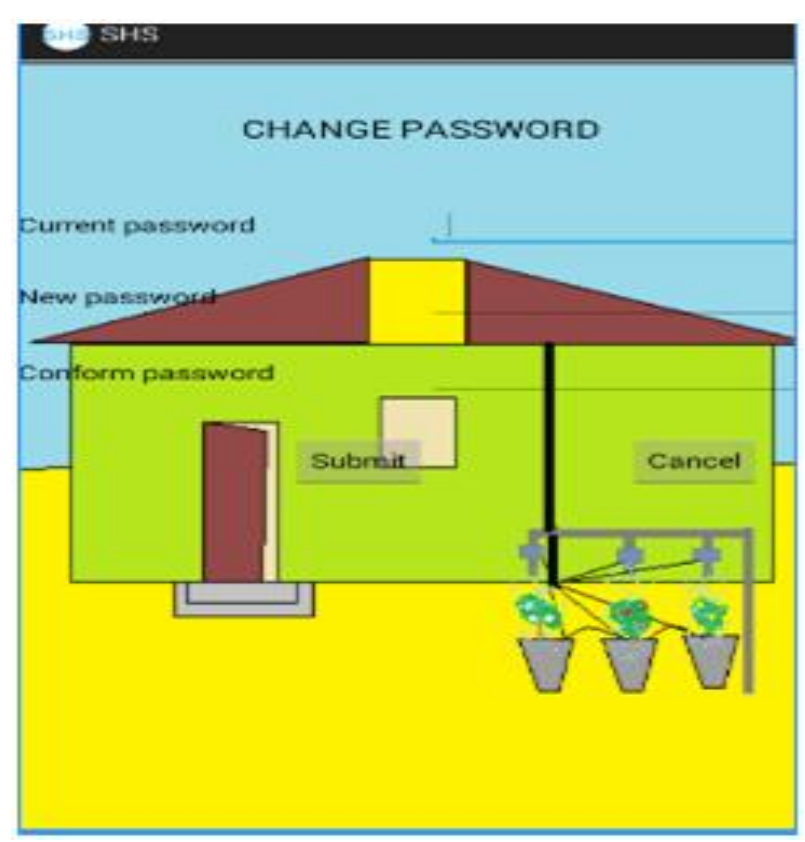

Fig 4.4: Different areas in the smart home to be controlled

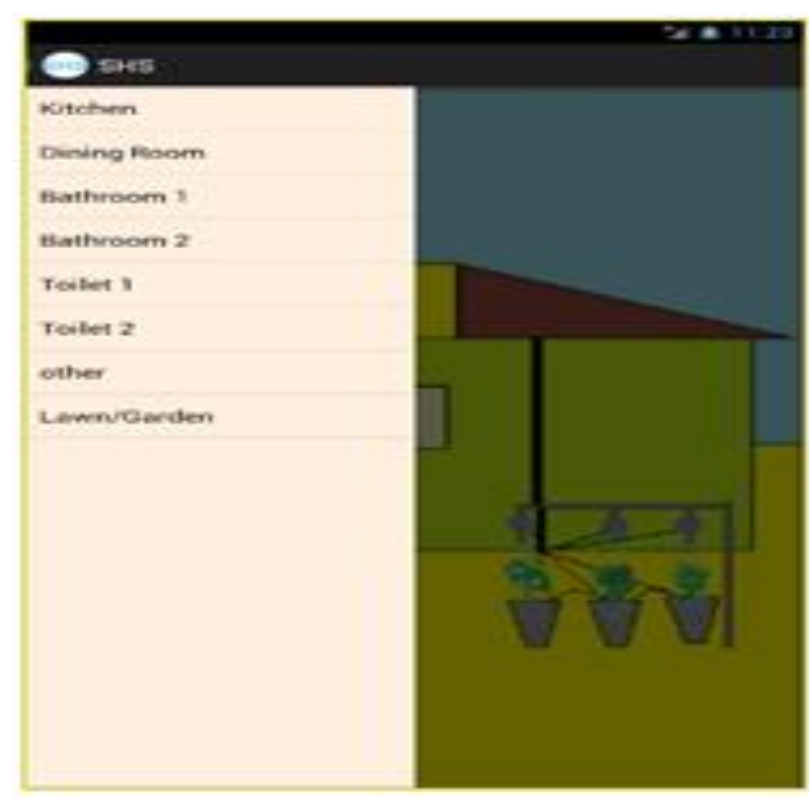

Fig 4.5 Password Change Option

\section{APPLICATIONS}

During holiday trips: This application could be used for watering the plants when somebody will go for a trip during holidays. Either the plants in the garden may dry because of no proper watering for many days or trouble has to be given to the neighbors for watering the plants. At this situation SHS app can be used and water the plant by checking the moisture level in the soil. Since it is controlled via internet it becomes easy to water the plants from any place.

Conservation of water: The situation may arise when the tap in the home might be on when there is no water in the tank and forgot to close it because of no water in the tank. Later when the tank gets water then there will be continuous leakage of water leading to wastage of water. At this situation the SHS app could be opened at any time and any place and if the status of the tap is in ON state, the tap can be closed. Using this app the wastage of water can be prevented.

\section{CONCLUSION AND FUTURE WORK}

The smart home application developed is a flexible and effective system which is helpful in everyday life especially for physically handicapped, patients and elderly people. It is also helpful in conservation of water, which can be efficiently implemented in gardens, parks etc. This application also provides the security as the valid user need to provide the authentication requirements in order to use it.

The smart home system application designed can be integrated with existing smart home systems so that it can be implemented in the large scale and more efficiently. This can be done my adding additional sensors and the SHS app to the existing system . Integrating this system to the camera based video surveillance will be of a great help to elderly people and patients. The camera based video surveillance helps to visually monitor the daily activities of elderly people and patients. After visually monitoring the activity this SHS app can be used to control the taps for the help of elderly people

\section{REFERENCES}

[1] Shiu Kumar, "Ubiquitous Smart Home System using Android Application" International Journal of Computer Networks \& Communications, vol. 6, No.1, January 2014.

[2] Mohamed Abd El-Latif Moawad, Ahmed Fathy, Ahmed Hafez "Smart Home Automated Control System using Android Application And Microcontroller" International Journal of Computer Networks \& Communications, vol. 5 , issue 5, may 2014

[3] S. V. Devika et al, Arduino Based Automatic Plant Watering System, International Journal of Advanced Research in Computer Science and Software Engineering ,pp. 449-456 (C) 2014, IJARCSSE Volume 4, Issue 10, October 2014 ISSN: 2277 128X

[4] Venkata Naga Rohit Gunturi Electronics and communication engineering department,Anna University,Chennai. Microcontroller Based Automatic Plant Irrigation System International Journal of Advancements in Research \& Technology, Volume 2, Issue4, April-2013 194 ISSN 2278-7763 Copyright (C) 2013 SciRes Pub. 